\title{
Sharp-interface model of electrodeposition and ramified growth
}

\author{
Nielsen, Christoffer Peder; Bruus, Henrik
}

Published in:

Physical Review E

Link to article, DOI:

10.1103/physreve.92.042302

Publication date:

2015

Document Version

Publisher's PDF, also known as Version of record

Link back to DTU Orbit

Citation (APA):

Nielsen, C. P., \& Bruus, H. (2015). Sharp-interface model of electrodeposition and ramified growth. Physical Review E, 92(4), [042302]. https://doi.org/10.1103/physreve.92.042302

\section{General rights}

Copyright and moral rights for the publications made accessible in the public portal are retained by the authors and/or other copyright owners and it is a condition of accessing publications that users recognise and abide by the legal requirements associated with these rights.

- Users may download and print one copy of any publication from the public portal for the purpose of private study or research.

- You may not further distribute the material or use it for any profit-making activity or commercial gain

- You may freely distribute the URL identifying the publication in the public portal

If you believe that this document breaches copyright please contact us providing details, and we will remove access to the work immediately and investigate your claim 


\title{
Sharp-interface model of electrodeposition and ramified growth
}

\author{
Christoffer P. Nielsen* and Henrik Bruus ${ }^{\dagger}$ \\ Department of Physics, Technical University of Denmark, DTU Physics Building 309, DK-2800 Kongens Lyngby, Denmark
}

(Received 26 June 2015; revised manuscript received 27 August 2015; published 5 October 2015)

\begin{abstract}
We present a sharp-interface model of two-dimensional ramified growth during quasisteady electrodeposition. Our model differs from previous modeling methods in that it includes the important effects of extended spacecharge regions and nonlinear electrode reactions. The electrokinetics is described by a continuum model, but the discrete nature of the ions is taken into account by adding a random noise term to the electrode current. The model is validated by comparing its behavior in the initial stage with the predictions of a linear stability analysis. The main limitations of the model are the restriction to two dimensions and the assumption of quasisteady transport.
\end{abstract}

DOI: 10.1103/PhysRevE.92.042302

PACS number(s): 82.45.Qr, 82.45.Gj, 61.43.Hv

\section{INTRODUCTION}

Electrodeposition is a technologically important process with diverse applications and implications, e.g., for battery technology, electroplating, and production of metal powders and microstructures [1-11]. For well over a century it has been known, however, that the layer deposited during electrodeposition is prone to morphological instabilities, leading to ramified growth of the electrode surface. Over the years, many experimental, theoretical, and numerical studies have been devoted to increasing the understanding of this ramified growth regime [12-20]. Big contributions to our understanding of the growth process have come from diffusion-limited aggregation (DLA) models [21,22] and, more recently, phasefield models similar to those that have successfully been applied to solidification problems [23-29]. However, while both of these approaches capture parts of the essential behavior of ramified growth, they have some fundamental shortcomings when applied to the electrodeposition problem.

The first of these shortcomings has to do with the ion transport in the system. Typically, the electrolyte contains a cation of the electrode metal that can both deposit on the electrodes and be emitted from the electrodes. The anion, on the other hand, is blocked by the electrodes. The electrodes thus act as ion-selective elements and for this reason the system exhibits concentration polarization when a voltage is applied. Smyrl and Newman showed [30] that in systems exhibiting concentration polarization, the linear ambipolar diffusion equation breaks down when the applied voltage exceeds a few thermal voltages. At higher voltages a nonequilibrium extended space-charge region develops next to the cathode, causing the transport properties of the system to change dramatically. It seems apparent that this change in transport properties must also lead to a change in electrode growth behavior. Indeed, this point was argued already by Chazalviel [12]. Now the issue with DLA and phase-field models is that neither of these methods account for nonzero space-charge densities. It is therefore only reasonable to apply these methods in the linear regime, where the applied voltage is smaller than a few thermal voltages.

\footnotetext{
*chnie@fysik.dtu.dk

†bruus@fysik.dtu.dk
}

The other shortcoming of DLA and phase-field methods is their treatment of the electrode-electrolyte interface. It is well known in electrochemistry that electrodeposition occurs with a certain reaction rate, which is dependent on the electrode overpotential and typically modeled using a Butler-Volmertype expression $[31,32]$. Thus, since the established reaction models are only defined for sharp interfaces, they cannot be properly implemented in DLA methods or in phase-field methods.

There have been attempts to include finite space-charge densities in phase-field models, but the resulting models are only practical for one-dimensional systems because they require an extremely dense meshing of the computational domain [23,24,33]. Attempts at including electrode reactions suffer from similar problems, as the proposed models are sensitive to the width of the interface region and to the interpolation function used in the interface region [26,34]. See Appendix A for a short discussion of this point.

To circumvent the shortcomings of the established models we pursue a different solution strategy in this paper. Rather than defining the interface via a smoothly varying timedependent parameter as in the phase-field models, we employ a sharp-interface model, in which the interface is moved for each discrete time step. Using a sharp-interface model has the distinct advantage that electrode reactions are easily implemented as boundary conditions. Likewise, it is fairly straightforward to account for nonzero space-charge densities in a sharp-interface model (see, for instance, our previous work in Refs. [35,36]).

Like most previous models, our sharp-interface model of electrodeposition models the electrode growth in two dimensions. There have been several experiments in which ramified growth is confined to a single plane and is effectually two dimensional [17,37-39]. However, for most systems ramified growth occurs in all three dimensions. There will obviously be some discrepancy between our two-dimensional (2D) results and the 3D reality, but we are hopeful that our 2D model does in fact capture much of the essential behavior.

At this stage, our sharp-interface model is only applicable once the initial transients in the concentration distribution have died out. In its current form the model is therefore mainly suitable for small systems, in which the diffusive time scale is reasonably small. We aim at removing this limitation in future work. 


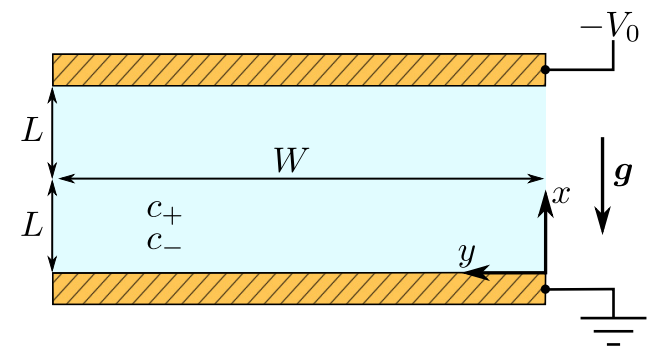

FIG. 1. (Color online) Sketch of the initial geometry of the system. Two coplanar metal electrodes of width $W$ are placed a distance of $2 L$ apart. The gap between them is filled by an electrolyte with cation concentration $c_{+}$and anion concentration $c_{-}$. A voltage difference of $V_{0}$ is applied between the electrodes.

\section{MODEL SYSTEM}

The model system consists of two initially flat parallel metal electrodes of width $W$ placed a distance of $2 L$ apart. The system is very thin in the third dimension, so the transport and growth can be modeled as quasi-two-dimensional. In the space between the electrodes is a binary symmetric electrolyte of concentration $c_{0}$, in which the cation is identical to the electrode material. The electrodes can thus act as both sources and sinks for the cation, whereas the anion can neither enter nor leave the system. A voltage difference $V_{0}$ (in units of the thermal voltage $V_{\mathrm{T}}=k_{\mathrm{B}} T / e$ ) is applied between the two electrodes, driving cations toward the top electrode and anions toward the bottom electrode. A sketch of the system is shown in Fig. 1.

By depositing onto the top electrode we ensure that the ion concentration increases from top to bottom, so we do not have to take the possibility of gravitational convection into account. Since the system is very thin, a similar convection suppression can be obtained by simply placing the $2 \mathrm{D}$ sample horizontally. To limit the complexity of the treatment, we also disregard any electro-osmotic motion, which may arise in the system. We note, however, that the sharp-interface model would be well suited to investigate the effects of electroosmosis since the space-charge density is an integral part of the model.

\section{SOLUTION METHOD}

The basic idea in our solution method is to solve the transport-reaction problem for each time step and then use the calculated currents to find the amount of material deposited at each point on the electrode. Based on this deposition rate, the geometry is updated and the transport-reaction problem is solved for a new time step, as illustrated in Fig. 2.

The major difficulty in employing this method is that when the geometry is updated the computational domain is also remeshed, so there is no straightforward way of continuing from the old solution of the transport-reaction problem. One way of getting around this issue is to separate the time scales in the problem. More to the point, we assume that the growth of the electrode happens so slowly, compared to the transport time scales, that the transport problem always is in a quasi-steady-state. By treating the transport-reaction problem as being in a steady state in each time step, a solution can be computed without reference to solutions at previous time steps.

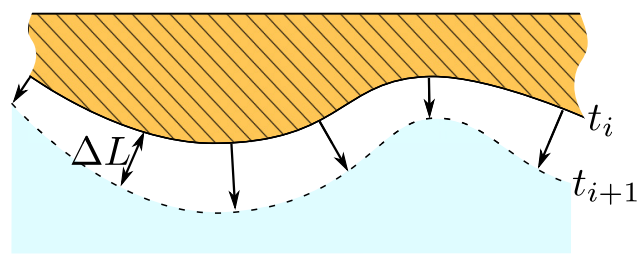

FIG. 2. (Color online) Sketch of the electrode growth. The electrode surface at time $t_{i}$ is indicated with a solid line. In the time step $t_{i+1}-t_{i}$ an amount of material $\Delta L$, which may vary with position, is deposited on the electrode. On the basis of the deposited material, the geometry at time $t_{i+1}$ is created (indicated with a dashed line).

Note that although the transport is modeled as quasisteady, the model is not Laplacian since we account for deviations from electroneutrality occurring at overlimiting current.

Obviously, the quasi-steady-state assumption is flawed in the initial time after a voltage is applied to the system, as the application of a voltage gives rise to some transients in the transport problem. However, after the initial transients have died out the assumption is quite reasonable, except for the case of very concentrated electrolytes. To see that, we consider the thickness $\Delta L$ of the electrode growth in a time interval $\Delta t$,

$$
\Delta L=a^{3} \Delta t J_{+}
$$

where $a^{3}$ is the volume of a metal atom in the solid phase and $J_{+}$is the current density of metal ions entering the electrode. The current density is on the order of the limiting current $2 c_{0} D_{+} / L$, so the time scale associated with an electrode growth of $\Delta L$ is

$$
\Delta t=\frac{\Delta L}{a^{3} J_{+}} \sim \frac{L \Delta L}{2 D_{+} c_{0} a^{3}} .
$$

On the other hand, the transport time scale $t_{\text {diff }}^{\Delta L}$ associated with the distance $\Delta L$ is

$$
t_{\text {diff }}^{\Delta L} \sim \frac{\Delta L^{2}}{2 D_{+}} .
$$

The ratio of the transport time scale to the growth time scale is thus

$$
\frac{t_{\mathrm{diff}}^{\Delta L}}{\Delta t} \sim \frac{\Delta L}{L} c_{0} a^{3},
$$

which is indeed very much smaller than unity.

As mentioned above, our model does not apply to the initial time after the voltage is applied. To estimate how this impacts our results, we make a comparison of the important time scales. The time it takes for the transients to die out is given by the diffusion time

$$
t_{\text {diff }}^{L}=\frac{L^{2}}{2 D_{+}} .
$$

The growth rate of the most unstable harmonic perturbation to the electrode surface is denoted by $\Gamma_{\max }$ (see Ref. [40]) and from this we obtain an instability time scale

$$
t_{\mathrm{inst}} \sim \frac{1}{\Gamma_{\max }} .
$$


It is apparent that if

$$
t_{\text {diff }}^{L} \lesssim t_{\text {inst }}
$$

then nothing interesting happens to the electrode surface in the time it takes the transients to disappear. In this case our quasisteady approach is therefore justified.

Even if $t_{\text {diff }}^{L} \gg t_{\text {inst }}$ our approach may be justified. If the total deposition time is much larger than $t_{\text {diff }}^{L}$, then what happens in the time before the transients die out is largely unimportant for the growth patterns observed in the end. Thus, though the quasisteady assumption seems restrictive, it actually allows us to treat a fairly broad range of systems.

\section{GOVERNING EQUATIONS}

\section{A. Bulk equations}

The ion-current densities in the system are given as

$$
\begin{aligned}
& \boldsymbol{J}_{ \pm}=-D_{ \pm} c_{0} c_{ \pm} \nabla \mu_{ \pm}, \\
& \mu_{ \pm}=\ln \left(c_{ \pm}\right)+z_{ \pm} \phi,
\end{aligned}
$$

where $D_{ \pm}$are the diffusivities of either ion, $c_{0}$ is the initial ion concentration, $c_{ \pm}$are the concentrations of either ion normalized by $c_{0}, \mu_{ \pm}$are the electrochemical potentials normalized by the thermal energy $k_{\mathrm{B}} T$, and $\phi$ is the electrostatic potential normalized by the thermal voltage $V_{\mathrm{T}}=k_{\mathrm{B}} T / e$. In the steady state the Nernst-Planck equations take the form

$$
0=-\nabla \cdot J_{ \pm} .
$$

The electrostatic part of the problem is governed by the Poisson equation

$$
2 \lambda_{\mathrm{D}}^{2} \nabla^{2} \phi=-\rho=-z_{+} c_{+}-z_{-} c_{-},
$$

where the Debye length $\lambda_{\mathrm{D}}$ is given as

$$
\lambda_{\mathrm{D}}=\sqrt{\frac{k_{\mathrm{B}} T \epsilon_{\mathrm{w}}}{2 e^{2} c_{0}}} .
$$

At the electrodes the anion flux vanishes,

$$
\boldsymbol{n} \cdot \boldsymbol{J}_{-}=0,
$$

and the cation flux is given by a reaction expression

$$
\boldsymbol{n} \cdot \boldsymbol{J}_{+}=-R \text {. }
$$

Rather than explicitly modeling the quasiequilibrium Debye layers at the electrodes, we follow Ref. [36] and implement a condition of vanishing cation gradient at the cathode

$$
\boldsymbol{n} \cdot \nabla c_{+}=0 .
$$

The last degree of freedom is removed by requiring global conservation of anions

$$
\int_{\Omega}\left(c_{-}-1\right) d V=0 .
$$

\section{B. Reaction expression}

We model the reaction rate using the standard ButlerVolmer expression [14]

$$
R=k_{0}\left[c_{+} e^{-\bar{\gamma} \kappa+\alpha Z(\phi+V)}-e^{-\bar{\gamma} \kappa-(1-\alpha) Z(\phi+V)}\right],
$$

where $k_{0}$ is the rate constant of the reaction, $V$ is the nondimensionalized electrode potential, $\kappa$ is the surface curvature, $\alpha$ is the charge-transfer coefficient, and $\bar{\gamma}$ is given in terms of the surface energy $\gamma$,

$$
\bar{\gamma}=\frac{a^{3} \gamma}{k_{\mathrm{B}} T} .
$$

Here $a^{3}$ is the volume occupied by one atom in the solid phase; $\bar{\gamma} \kappa$ is thus a measure of the energy per atom relative to the thermal energy. In Appendix A we discuss how the sharp-interface reaction model (16) relates to typical phasefield reaction models.

\section{NUMERICAL STABILITY}

Due to the surface energy term in the reaction expression, the surface is prone to numerical instability. In an attempt to reach the energetically favorable surface shape, the solver will sequentially overshoot and undershoot the correct solution. The fundamental issue we are facing is that the problem at hand is numerically stiff. As long as we are using an explicit time-integration method we are therefore likely to encounter numerical instabilities.

\section{A. Updating the interface position}

The straightforward way of updating the position $r$ of the interface is to use the explicit Euler method

$$
\boldsymbol{r}(t+\Delta t)=\boldsymbol{r}(t)+\boldsymbol{n} a^{3} \Delta t R(t)
$$

where $R(t)$ is the (position-dependent) reaction rate at time $t$. To avoid numerical instabilities, we should instead use the implicit Euler method

$$
\boldsymbol{r}(t+\Delta t)=\boldsymbol{r}(t)+\boldsymbol{n} a^{3} \Delta t R(t+\Delta t)
$$

where the reaction rate is evaluated at the end point instead of at the initial point; however, this is easier said than done. Here $R(t+\Delta t)$ depends on $\boldsymbol{r}(t+\Delta t)$ as well as on the concentration and potential distribution at $t+\Delta t$. Even worse, through the curvature $R(t+\Delta t)$ also depends on the spatial derivatives of $\boldsymbol{r}(t+\Delta t)$.

The way forward is to exploit that only part of the physics gives rise to numerical instabilities. It is therefore sufficient to evaluate the problematic surface energy at $t+\Delta t$ and evaluate the remaining terms at $t$. For our purposes we can therefore make the approximation

$$
R(t+\Delta t) \approx R(t, \kappa(t+\Delta t)),
$$

where $\kappa$ is the curvature. This still makes for a quite complicated nonlinear partial differential equation (PDE), but we are getting closer to something tractable. The difference in curvature between $t$ and $t+\Delta t$ is small (otherwise we are taking too big time steps), so we can approximate

$$
R(t, \kappa(t+\Delta t)) \approx R(t, \kappa(t))+R^{\prime}(t, \kappa(t)) \Delta \kappa,
$$

where $R^{\prime}$ denotes $R$ differentiated with respect to $\kappa$ and $\Delta \kappa=$ $\kappa(t+\Delta t)-\kappa(t)$. The curvature can be written as

$$
\kappa=\frac{\partial \theta}{\partial s},
$$


where $\theta$ is the tangential angle of the interface and $s$ is the arc length along the interface. We therefore have

$$
\Delta \kappa=\kappa(t+\Delta t)-\kappa(t)=\frac{\partial \theta_{2}}{\partial s_{2}}-\frac{\partial \theta_{1}}{\partial s_{1}},
$$

where we have adopted the shorthand notation 1 and 2 for times $t$ and $t+\Delta t$, respectively. The arc lengths $s_{1}$ and $s_{2}$ will obviously differ for any nonzero displacement, but this is a small effect compared to the angle difference. As an approximation we therefore use $s_{2} \approx s_{1}$ and obtain

$$
\Delta \kappa \approx \frac{\partial\left(\theta_{2}-\theta_{1}\right)}{\partial s_{1}} .
$$

The tangential angle is a function of the surface parametrization

$$
\tan \left(\theta_{1}\right)=\frac{\partial y_{1}}{\partial x_{1}}
$$

For small displacements we can approximate

$$
\begin{aligned}
\tan \left(\theta_{2}\right) & =\frac{\partial y_{2}}{\partial x_{2}}=\frac{\partial\left(y_{1}+\Delta y\right)}{\partial\left(x_{1}+\Delta x\right)} \\
& \approx \tan \left(\theta_{1}\right)+\frac{\partial \Delta y}{\partial x_{1}}-\tan \left(\theta_{1}\right) \frac{\partial \Delta x}{\partial x_{1}} .
\end{aligned}
$$

The difference in tangential angles can then be written

$$
\begin{aligned}
\theta_{2}-\theta_{1} & =\arctan \left[\tan \left(\theta_{1}\right)+\frac{\partial \Delta y}{\partial x_{1}}-\tan \left(\theta_{1}\right) \frac{\partial \Delta x}{\partial x_{1}}\right]-\theta_{1} \\
& \approx \frac{1}{1+\tan ^{2}\left(\theta_{1}\right)}\left[\frac{\partial \Delta y}{\partial x_{1}}-\tan \left(\theta_{1}\right) \frac{\partial \Delta x}{\partial x_{1}}\right]
\end{aligned}
$$

Returning to the implicit Euler method (19), we project it onto the normal vector to obtain

$$
\begin{aligned}
\Delta L & =a^{3} \Delta t R(t+\Delta t) \\
& \approx a^{3} \Delta t\left[R(t, \kappa(t))+R^{\prime}(t, \kappa(t)) \Delta \kappa\right],
\end{aligned}
$$

where $\Delta L=\boldsymbol{n} \cdot[\boldsymbol{r}(t+\Delta t)-\boldsymbol{r}(t)]$. The increments in the $x$ and $y$ directions are related to $\Delta L$ via

$$
\Delta x=n_{x} \Delta L, \quad \Delta y=n_{y} \Delta L .
$$

Inserting these in Eq. (27) and writing out the curvature difference $\Delta \kappa$, we obtain a linear PDE for the displacement $\Delta L$

$$
\frac{\Delta L-a^{3} \Delta t R(t, \kappa(t))}{a^{3} \Delta t R^{\prime}(t, \kappa(t))}=\Delta \kappa=\frac{\partial}{\partial s_{1}}\left\{\frac{n_{y}-n_{x} \tan \left(\theta_{1}\right)}{1+\tan ^{2}\left(\theta_{1}\right)} \frac{\partial \Delta L}{\partial x_{1}}\right\} .
$$

In the limit $\Delta \kappa=0$ this equation reduces to the original forward Euler method (18).

\section{B. Correction for the curvature}

In the previous derivation, we did not take into account that the local curvature slightly changes the relation between amount of deposited material and surface displacement $\Delta L$. The deposited area in an angle segment $d \theta$ can be calculated as

$d A=\frac{d \theta}{2 \pi}\left[\pi\left(\frac{1}{\kappa}+\Delta L\right)^{2}-\pi \frac{1}{\kappa^{2}}\right]=\frac{d \theta}{2}\left[\Delta L^{2}+2 \frac{\Delta L}{\kappa}\right]$.

The line segment $d s$ is related to the angle segment as $d s=$ $d \theta / \kappa$. This means that

$a^{3} \Delta t R(t+\Delta t)=\frac{d A}{d s}=\frac{\kappa}{2}\left[\Delta L^{2}+2 \frac{\Delta L}{\kappa}\right]=\Delta L+\frac{\kappa}{2} \Delta L^{2}$.

Using this expression in Eq. (28) yields the slightly nonlinear PDE, with the term $\frac{1}{2} \kappa \Delta L^{2}$,

$$
\begin{gathered}
\frac{\Delta L+(\kappa / 2) \Delta L^{2}-a^{3} \Delta t R(t, \kappa(t))}{a^{3} \Delta t R^{\prime}(t, \kappa(t))} \\
=\frac{\partial}{\partial s_{1}}\left\{\frac{n_{y}-n_{x} \tan \left(\theta_{1}\right)}{1+\tan ^{2}\left(\theta_{1}\right)} \frac{\partial \Delta L}{\partial x_{1}}\right\},
\end{gathered}
$$

in place of Eq. (30).

\section{NOISE}

An important part of the problem is the noise in the system, since the noise is what triggers the morphological instability and leads to the formation of dendrites. Exactly how the noise should be defined, however, is a matter of some uncertainty. Most previous work uses a thermal white noise term with a small but seemingly arbitrary amplitude. In this work we use a slightly different approach, in which we assume that the noise is entirely attributed to shot noise.

As it turns out, this approach requires us to be more specific about how our 2D model is related to the three-dimensional reality. In Fig. 3 a sketch of the three-dimensional electrode is shown. The electrode interface is free to vary in the $x y$ plane, but has a fixed depth $\Delta h$ in the $z$ direction. Obviously, most real electrodeposits will have a more complicated behavior in the $z$ direction, but for electrodeposits grown in a planar confined geometry this is actually a reasonable description.

Solving the transport-reaction problem yields the current density at each point along the electrode surface, which is the average number of ions arriving per surface area per time. The mean number $Q$ of ions arriving in an electrode section of size $\Delta h \Delta s$ in a time interval $\Delta t$ is thus

$$
Q=J_{+} \Delta h \Delta s \Delta t
$$

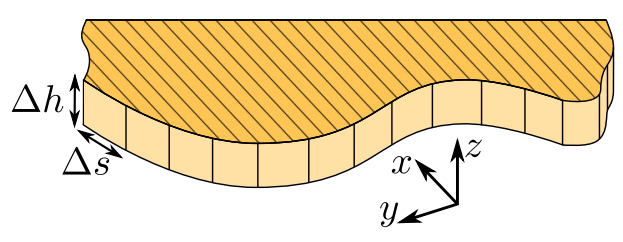

FIG. 3. (Color online) Three-dimensional extension of our twodimensional model. The electrode interface can vary in the $x y$ plane according to the calculated ion currents, but it has a fixed depth $\Delta h$ in the $z$ direction. The interface is also divided into a number of bins of width $\Delta s$ in the $x y$ plane. Each bin thus has the area $\Delta h \Delta s$. 
Since the ions are discrete entities, the actual number of arriving ions will, however, fluctuate randomly around the mean $Q$ with some spread $\sigma$. We assume that within the time interval $\Delta t$, the arrival of each ion is statistically uncorrelated with the arrival of each other ion. It can then be shown that, as long as $Q \gtrsim 10$, the number of arriving ions follows a normal distribution with mean $Q$ and standard deviation

$$
\sigma=\sqrt{Q}
$$

This corresponds to an extra random current density

$$
J_{\text {rand }}=\frac{\sqrt{Q}}{\Delta h \Delta s \Delta t} q_{\mathrm{rand}}=\sqrt{\frac{J_{+}}{\Delta h \Delta s \Delta t}} q_{\mathrm{rand}},
$$

where $q_{\text {rand }}$ is a random number taken from a normal distribution with mean 0 and standard deviation 1 . This in turn corresponds to a random electrode growth of

$$
\Delta L_{\mathrm{rand}}=a^{3} \sqrt{\frac{J_{+} \Delta t}{\Delta h \Delta s}} q_{\mathrm{rand}} .
$$

Now there is something slightly unusual about this expression for the random growth: It seems that the random growth becomes larger the smaller the bin size $\Delta s$ is. However, as the bin size becomes smaller the weight of that bin in the overall behavior is also reduced. The net effect is that the bin size $\Delta s$ does not matter for the random growth (see Appendix B for a more thorough treatment).

The bin depth $\Delta h$, on the other hand, does matter for the random growth. Since our model is not concerned with what happens in the $z$ direction, we simply have to choose a physically reasonable value of $\Delta h$ and accept that our choice will have some impact on the simulations. This is an unfortunate consequence we must accept for applying a $2 \mathrm{D}$ model to a $3 \mathrm{D}$ phenomenon.

\section{NUMERICAL SOLUTION}

To solve the electrodeposition problem we use the commercially available finite-element software COMSOL MULTIPHYSICS version 4.3a together with MATLAB version 2013b. Following our previous work $[35,36,41]$, the governing equations and boundary conditions (8)-(10), (12)-(16), and (33) are rewritten in weak form and implemented in the mathematics module of COMSOL. For each time step, the following steps are carried out. First, a list of points defining the current electrode surface is loaded into COMSOL and the surface is created using a cubic spline interpolation between the given points. The computational domain is meshed using a mesh size of $\Delta s$ at the electrode surface, a mesh size of $l$ in a small region next to the electrode, and a much coarser mesh in the remainder of the domain. Next, the curvature of the surface is calculated at each point. The solution from the previous time step is then interpolated onto the new grid to provide a good initial guess for the transport-reaction problem. Then the transport-reaction problem is solved. Based on the solution to the transport-reaction problem, the electrode growth $\Delta L$ is calculated by solving Eq. (33) on the electrode boundary. At each mesh point a small random contribution $\Delta L_{\text {rand }}=a^{3} \Delta t J_{\text {rand }}$ is then added to $\Delta L$. Finally, the new $x$ and $y$ positions are calculated by adding $n_{x}\left(\Delta L+\Delta L_{\text {rand }}\right)$ and $n_{y}\left(\Delta L+\Delta L_{\text {rand }}\right)$ to the old $x$ and $y$ positions.
The new $x$ and $y$ positions are exported to MATLAB. In MATLAB any inconsistencies arising from the electrode growth are resolved. If, for instance, the electrode surface intersects itself, the points closest to each other at the intersection position are merged and any intermediate points are discarded. This corresponds to creating a hollow region in the electrode that is no longer in contact with the remaining electrolyte. The points are then interpolated so that they are evenly spaced and exported to COMSOL so that the entire procedure can be repeated for a new time step.

The simulations are run on a standard work station with two 2.67-GHz Intel Xeon processors and 48-GB RAM. The electrodeposits shown in Sec. VIII typically take 2 days to run.

\section{A. Reduction of the computational domain}

At the cathode the mesh is much finer than in the remainder of the domain. The number of mesh points, and hence the computation time, therefore roughly scales with the length of the electrolyte-cathode interface. This has the unfortunate consequence that the computation time for each time step increases drastically, when branching structures emerge at the cathode. To lower the computation time we exploit the fact that the vast majority of the current enters near the tips of the dendritic structures. The parts of the cathode that are not near the tips can therefore be left fixed in time and thus removed from the simulation, without changing the results appreciably. This part of the domain is called the passive region. In regions where the current density is less than 0.001 times the maximum value, we thus substitute the real ramified electrode with a smooth line connecting the parts of the electrode with larger currents. The procedure is carried out in such a way that the real electrode surface can always be recovered from the reduced surface. For a few select examples we have verified that the results are virtually unchanged by this simplifying procedure. One of these examples is shown in Appendix C. Shown in Fig. 4 is an example of an electrode surface together

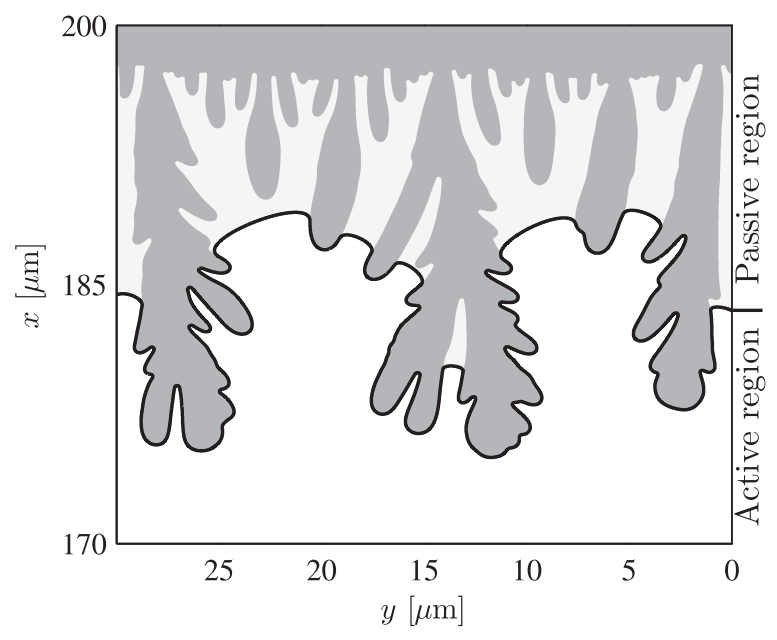

FIG. 4. Example of the simplifying cutting procedure. The reduced interface (thick black line) divides the domain into an active region (white) and a passive region (light gray). The dark gray area shows the real cathode. The example is taken from a simulation with $c_{0}=1 \mathrm{mM}$ and $V_{0}=10$ after deposition for $31 \mathrm{~h}$ and $28 \mathrm{~min}$. 
TABLE I. Fixed parameter values used in the simulations.

\begin{tabular}{lcl}
\hline \hline Parameter & Symbol & \multicolumn{1}{c}{ Value } \\
\hline cation diffusivity $^{\mathrm{a}}$ & $D_{+}$ & $0.714 \times 10^{-9} \mathrm{~m}^{2} \mathrm{~s}^{-1}$ \\
anion diffusivity $^{\mathrm{a}}$ & $D_{-}$ & $1.065 \times 10^{-9} \mathrm{~m}^{2} \mathrm{~s}^{-1}$ \\
ion valence $_{\text {surface energy }}$ & $\gamma$ & 2 \\
temperature $_{\text {permittivity of water }}$ & $T$ & $1.85 \mathrm{~J} / \mathrm{m}^{2}$ \\
charge-transfer coefficient $^{\text {reaction constant }}$ & $\epsilon_{\mathrm{w}}$ & $6.90 \times 10^{-10} \mathrm{~F} / \mathrm{m}$ \\
diameter of a copper atom $^{\mathrm{c}}$ & $\alpha$ & 0.5 \\
\hline
\end{tabular}

${ }^{\text {a Reference [42]. }}$

${ }^{\mathrm{b} C a l c u l a t e d ~ u s i n g ~ t h e ~ e x c h a n g e ~ c u r r e n t ~} I_{0}=30 \mathrm{~A} / \mathrm{m}^{2}$ from Ref. [43] and $k_{0}=I_{0} / Z e$.

${ }^{\mathrm{c}}$ The cubic root of the volume per atom in solid copper [42].

with the reduced surface. It can be seen that the length of the electrolyte-cathode interface is heavily reduced by excluding parts of the electrode from the computation.

\section{B. Parameter values}

To limit the parameter space we choose fixed, physically reasonable values for the parameters listed in Table I. The values are chosen to correspond to copper electrodes in a copper sulfate solution (see Ref. [40] for details).

In Ref. [40] we calculate the critical wavelength $\lambda_{c}$, i.e., the smallest unstable perturbation wavelength, for a range of parameters. See Appendix D for a brief outline of the results of the stability analysis. We expect the critical wavelength to be the smallest feature in the problem, so we choose the mesh size accordingly. We set the mesh size at the electrode to $\Delta s=0.1 \lambda_{\mathrm{c}}$ since our investigations (see Sec. VII C) show that this is a suitable resolution. We also require that the mesh size does not exceed 0.1 times the local radius of curvature. In the bulk part of the system we use a relatively coarse triangular mesh with mesh size $W / 6$. Close to the cathode, in a region $l=0.5 \mu \mathrm{m}$ from the electrode surface, we use a triangular mesh with mesh size $l / 4$. See Fig. 5 for a meshing example.

We choose a fixed value for the bin depth $\Delta h=0.2 \lambda_{\mathrm{c}}$. In accord with the analysis in Appendix $\mathrm{B}$, the time step $\Delta t$ is chosen so that it is always smaller than $0.5 / \Gamma_{\max }$. In addition, the time step is chosen so that at each point on the cathode the growth during the time step is smaller than the local radius of curvature.

We fix the length $L$ to $100 \mu \mathrm{m}$. According to the time-scale analysis in Sec. III and the instability growth rates found in Ref. [40], the quasi-steady-state approximation is valid for $L=100 \mu \mathrm{m}$. The width $W$ of the system is set to $W=200 \lambda_{\text {c }}$, rounded to the nearest micrometer. This makes for a system that is broad enough to exhibit interesting growth patterns, while having a reasonable computation time. The growth is somewhat affected by the symmetry boundaries at $y=0$ and $y=W$, especially at later times.

These choices leave us with two free parameters, which are the bias voltage $V_{0}$ and the electrolyte concentration $c_{0}$. We solve the system for $c_{0}=\{1 \mathrm{mM}, 10 \mathrm{mM}, 100 \mathrm{mM}\}$ and $V_{0}=\{10,20,30\}$.

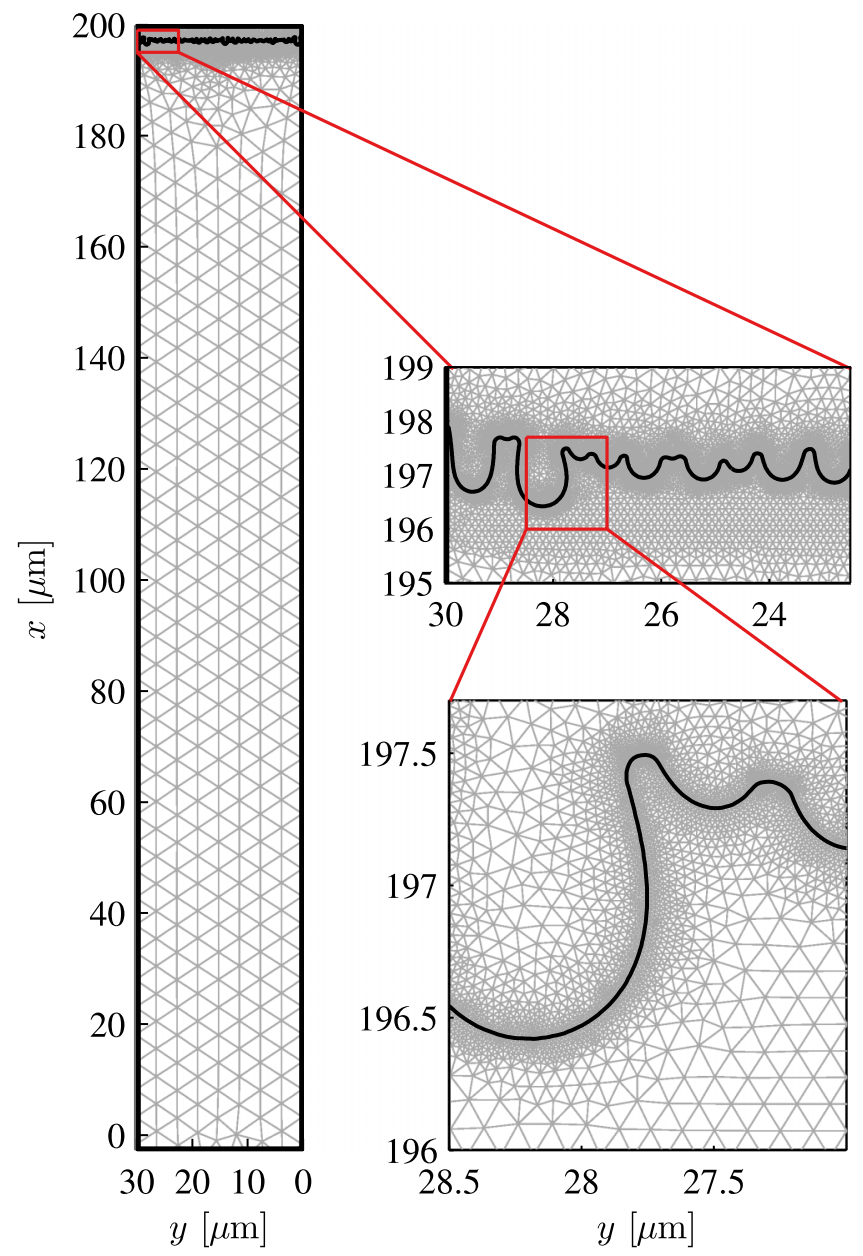

FIG. 5. (Color online) Example of domain meshing at varying magnification. The example is taken from a simulation with $c_{0}=$ $1 \mathrm{mM}$ and $V_{0}=10$ after deposition for $7 \mathrm{~h}$ and $50 \mathrm{~min}$. The wiggly black line is the cathode surface. The light gray lines are the mesh boundaries and the dark (red) lines show the sections that are magnified. The mesh elements above the cathode surface are only used for storing the solution between time steps.

\section{Validation}

The random nature of the phenomena we are investigating poses obvious challenges when it comes to validating the numerical simulations. The individual steps in the computation can be, and have been, thoroughly tested and validated, but testing whether the aggregate behavior after many time steps is correct is a much more difficult task. At some level, we simply have to trust that, if the individual steps are working correctly, then the aggregate behavior is also correct. To support this view, there is one test we can make of the aggregate behavior in the very earliest part of the simulation.

In the early stages of the simulation the electrode surface is deformed so little that the linear stability analysis from [40] should still be valid. We thus have an analytical expression for the wavelength-dependent growth rate $\Gamma$, which we can compare with the growth rates found in the numerical simulations. In Appendix B we calculate an expression for the average power spectrum of the cathode interface after deposition for a time $t_{\text {tot }}$, given the type of noise described 


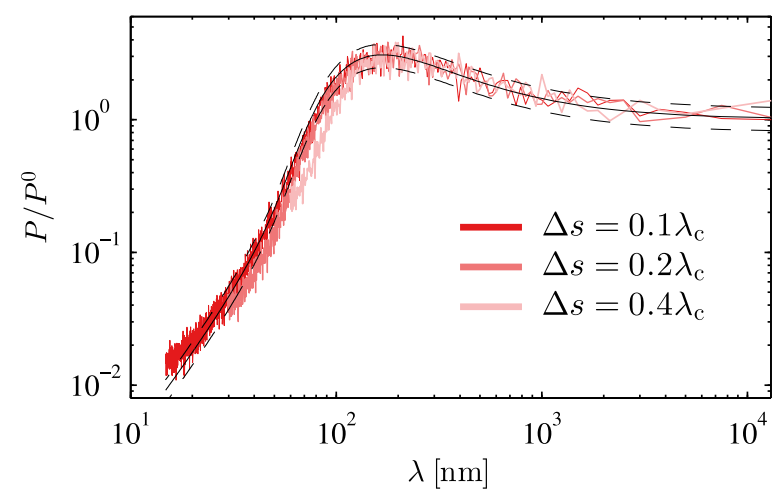

FIG. 6. (Color online) Power spectra averaged over 50 runs for three different mesh sizes $\Delta s=\left\{0.1 \lambda_{\mathrm{c}}, 0.2 \lambda_{\mathrm{c}}, 0.4 \lambda_{\mathrm{c}}\right\}$. In each run we used $M=100$ time steps of $\Delta t=0.64 \mathrm{~s}$ and the parameter values $c_{0}=10 \mathrm{mM}, L=100 \mu \mathrm{m}$, and $V_{0}=30$. The solid black line shows the analytical result and the dashed black lines show the analytical standard error on the mean. The result for $\Delta s=0.1 \lambda_{\mathrm{c}}$ is shown in dark gray (red), the result for $\Delta s=0.2 \lambda_{\mathrm{c}}$ is shown in medium gray (red), and the result for $\Delta s=0.4 \lambda_{\mathrm{c}}$ is shown in light gray (red).

in Sec. VI,

$$
\left\langle P_{n}\right\rangle=a^{6} \frac{J_{+}}{2 \Delta h W \Gamma_{n}}\left[e^{2 \Gamma_{n} t_{\mathrm{tot}}}-1\right],
$$

where $\Gamma_{n}$ is the growth rate of the $n$th wavelength $\lambda_{n}=W / n$ component in the noise spectrum. We also find the standard deviation $\operatorname{SD}\left(P_{n}\right)$ of the power spectrum

$$
\mathrm{SD}\left(P_{n}\right) \approx \sqrt{2}\left\langle P_{n}\right\rangle
$$

Because the standard deviation of $P_{n}$ is so large compared to the mean value, it is necessary to average over many runs before a meaningful comparison with Eq. (38) can be made. Averaging the power spectrum over 50 simulations brings the standard error on the mean down to $20 \%$ times the mean value, at which point a reasonable comparison can be made. In Fig. 6 the power spectrum averaged over 50 runs is shown for three different mesh sizes $\Delta s=\left\{0.1 \lambda_{c}, 0.2 \lambda_{c}, 0.4 \lambda_{c}\right\}$. In each run we used $M=100$ time steps of $\Delta t=0.64 \mathrm{~s}$ and the parameter values $c_{0}=10 \mathrm{mM}, L=100 \mu \mathrm{m}$, and $V_{0}=30$. The chosen step size corresponds to $0.01 / \Gamma_{\max }$. The analytical result (38) is also shown together with the standard error on the mean. The power spectra are normalized with the power $P^{0}$ obtained for $\Gamma=0$,

$$
P^{0}=a^{6} \frac{J_{+} t_{\mathrm{tot}}}{\Delta h W}
$$

It can be seen that for $\Delta s=0.4 \lambda_{\mathrm{c}}$ some of the power in the small-wavelength components is filtered out. As the mesh size is decreased to $\Delta s=0.2 \lambda_{\mathrm{c}}$ and $0.1 \lambda_{\mathrm{c}}$ the low-wavelength components are represented increasingly well.

In the above treatment, the time step was chosen very small compared to the instability time scale $\Delta t=0.01 / \Gamma_{\max }$. This was done to approach the limit of continuous time

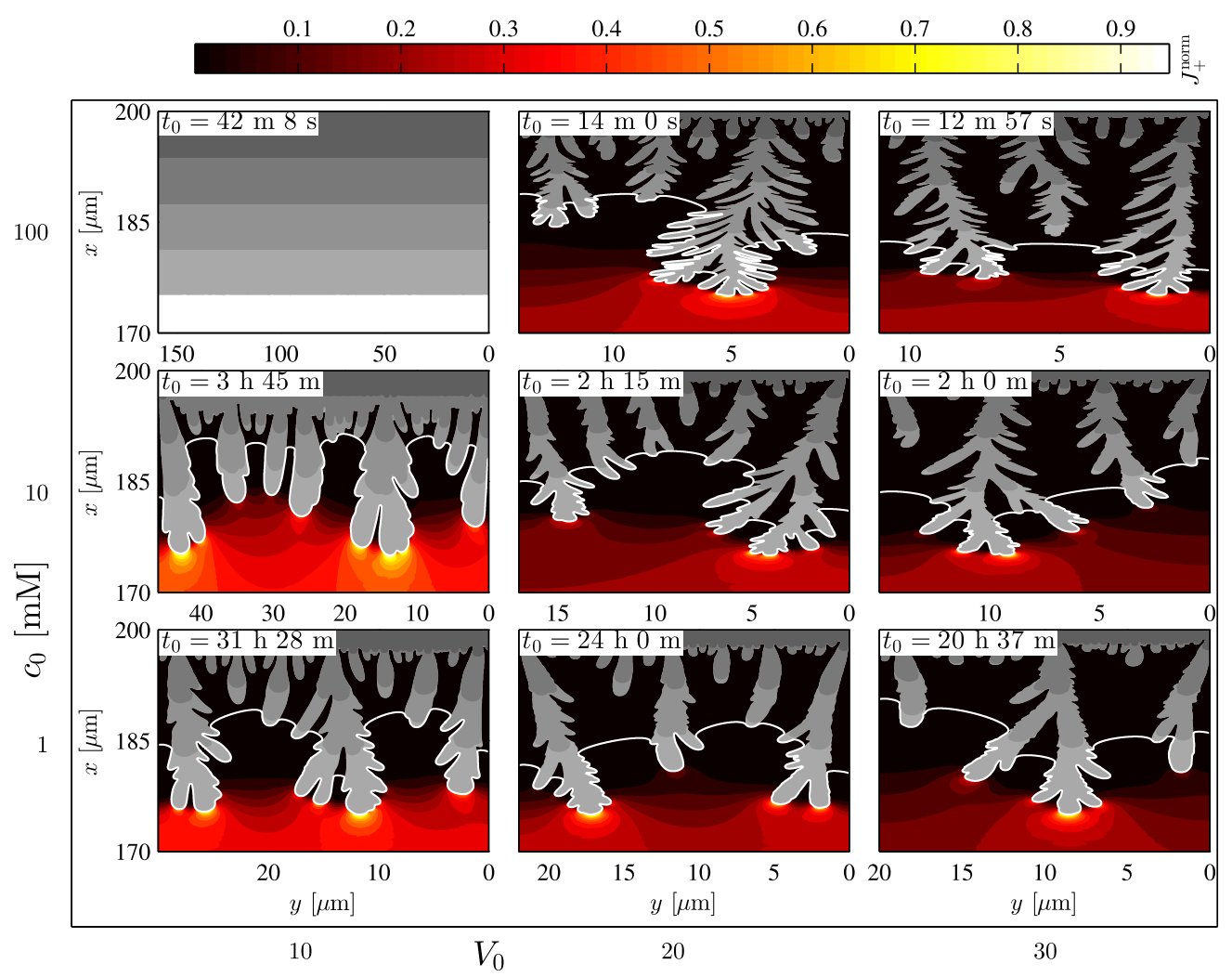

FIG. 7. (Color online) Electrodeposits in the $V_{0}-c_{0}$ plane obtained for $L=100 \mu \mathrm{m}, \quad c_{0}=\{1 \mathrm{mM}, 10 \mathrm{mM}, 100 \mathrm{mM}\}$, and $V_{0}=$ $\{10,20,30\}$. The aspect ratio varies between the panels, since the width $W$ of the simulated region is always set to $200 \lambda_{\mathrm{c}}$. The gray area has different shades corresponding to times $t_{0}$ (light), $0.75 t_{0}$ (darker), $0.5 t_{0}$ (darker yet), $0.25 t_{0}$ (darkest). The white line indicates the reduced surface at time $t_{0}$. The contours in the liquid represent the relative magnitude of the cation current. 


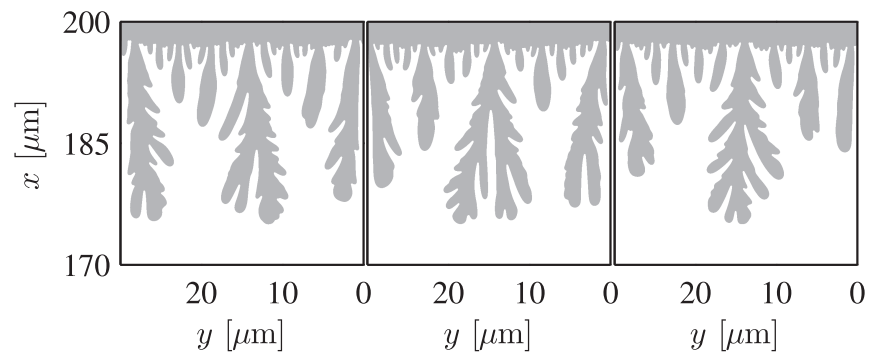

FIG. 8. Three simulations of electrodeposits using the same parameter values $L=100 \mu \mathrm{m}, c_{0}=1 \mathrm{mM}$, and $V_{0}=10$. The electrodeposits are clearly different from one another, but they do share some general features.

and thus enable the best possible comparison with the analytical theory. Such a short time step, however, is impractical for the much longer simulations in the remainder of the paper. In those simulations we use time steps as large as $\Delta t=0.5 / \Gamma_{\max }$. Due to the coarser time resolution employed in the remaining simulations, we expect their power spectrum to deviate somewhat from the almost ideal behavior shown in Fig. 6.

\section{RESULTS}

We let the simulations run until the cathode has grown $25 \mu \mathrm{m}$. The time $t_{0}$ it takes to reach this point varies greatly with the parameters, mainly because the limiting current scales with $c_{0}$. In Fig. 7 the cathode surfaces are shown along with heat plots showing the relative magnitude of the current density at the last time step. The white line shows the position of the reduced interface at the last time step and the gray area shows the actual position and shape of the cathode. The gray electrodeposits have different shades corresponding to $0.25 t_{0}, 0.5 t_{0}, 0.75 t_{0}$, and $t_{0}$. The heat plot shows the value of $J_{+}^{\text {norm }}$, which is the magnitude of the cation current density normalized with its maximum value. In each panel $J_{+}^{\text {norm }}$ thus varies from 0 to 1 .

To investigate the reproducibility of the results we have repeated the simulation of the $c_{0}=1 \mathrm{mM}$ and $V_{0}=10$ system two times. All three electrodeposits are shown in Fig. 8. The electrodeposits are clearly different from one another, as expected for a random process, but they are also seen to share some general features. These shared features are most easily appreciated by comparing the electrodeposits in Fig. 8 to the electrodeposits in Fig. 7. It can be seen that the electrodeposits in Fig. 8 are much more similar to each other than to any of the remaining electrodeposits in Fig. 7. Thus, the results are reproducible in the sense that the random electrodeposits have some general features that are determined by the parameter values.

When interpreting the plots in Fig. 7 we should be mindful that the aspect ratio is not the same in each panel. The reason for this is that the vertical axis has the same length, $30 \mu \mathrm{m}$, in each panel, while the length of the horizontal axis $W$ varies between panels. In Fig. 9 we show adapted versions of the panels from Fig. 7. The subfigures in Fig. 9 are created by repeatedly mirroring the subfigures from Fig. 7 until their horizontal length is $100 \mu \mathrm{m}$. Obviously, the resulting extended cathodes are somewhat artificial since we have imposed some symmetries that would not be present in a simulation of a system with $W=100 \mu \mathrm{m}$. Nevertheless, we find the subfigures in Fig. 9 useful since they give a rough impression of the appearance of wider systems and allow for easier comparison of length scales between panels.

\section{Rationalizing the cathode morphologies}

The cathode morphologies observed in Figs. 7 and 9 are a function of several factors, some of which we attempt to outline below. First, we consider the time $t_{0}$ it takes before part of the cathode reaches $x=175 \mu \mathrm{m}$. As can be seen from Eq. (1), this time is mainly a function of the limiting current.

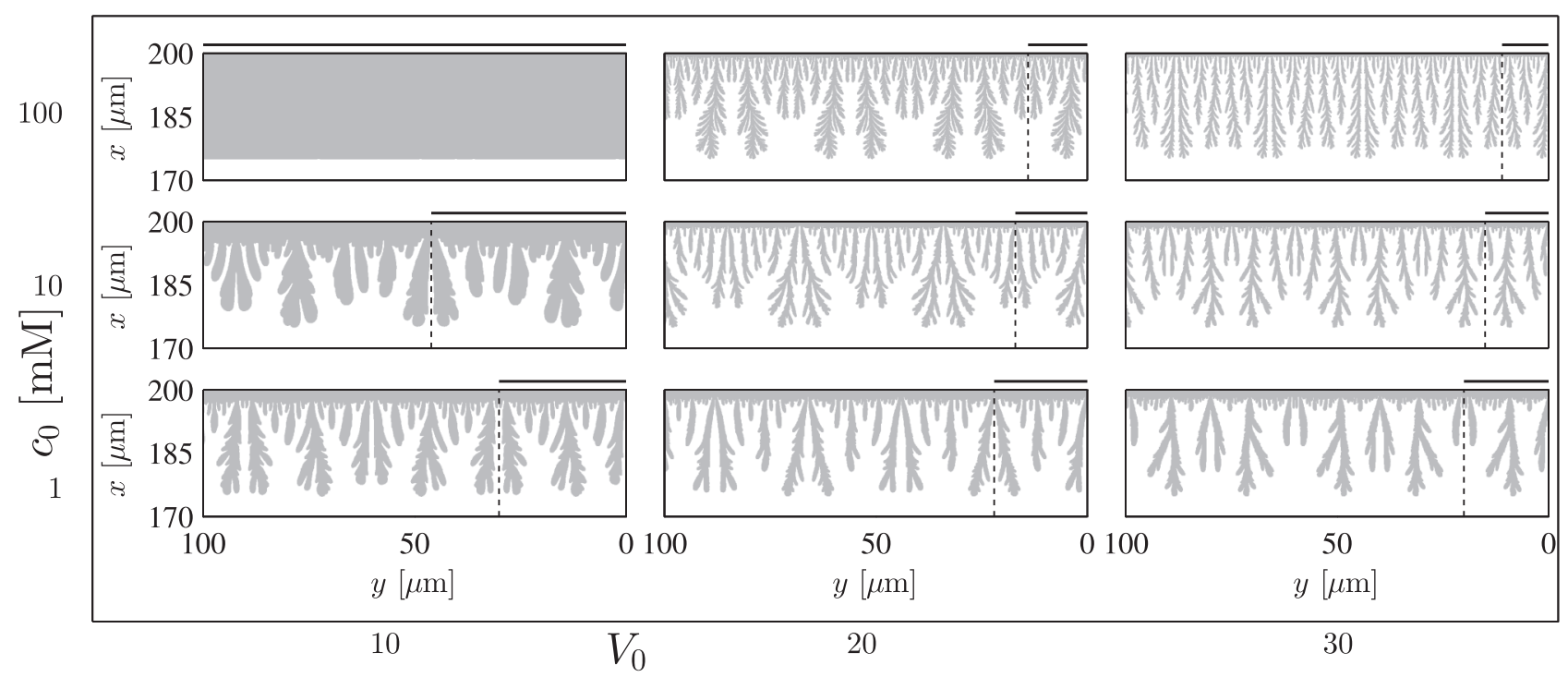

FIG. 9. Electrodeposits in the $V_{0}-c_{0}$ plane extended to an equal width of $100 \mu \mathrm{m}$ by mirror repetition of the original part from Fig. 7 (marked by the horizontal black line) between $0 \mu \mathrm{m}$ and the first mirror axis (vertical dashed line). Here, in contrast to Fig. 7, the aspect ratio is constant across the panels. 
This explains the approximately inverse scaling with $c_{0}$. The current density also increases with $V_{0}$, which is why the time $t_{0}$ decreases slightly as $V_{0}$ increases. Finally, the time $t_{0}$ scales with the filling factor. This is the reason why $t_{0}$ is much larger in the upper left panel of Fig. 7 than in either of the two other top row panels.

It is apparent from the lack of ramified growth that the cathode in the upper left panel in Fig. 7 is considerably more stable than the other systems in the leftmost column. To explain this variation in stability, we refer to Fig. 6 in Ref. [40]. There it is shown that the instability length scale is on the order of $50 \mu \mathrm{m}$ for $c_{0}=100 \mathrm{mM}$ at $V_{0}=10$, while it is considerably lower for $c_{0}=10$ and $1 \mathrm{mM}$. Figure 6 in Ref. [40] also shows that for $V_{0}>18$ the instability length scale decreases in size as the concentration increases. The same tendency is observed in Fig. 9.

From the subfigures in Fig. 9 it appears that there is a connection between the thickness $\delta_{\text {inst }}$ of the layer deposited before the instabilities develop (the base layer between $200 \mu \mathrm{m}$ and the onset of the ramification) and the characteristic length scale $\delta_{\text {char }}$ of the ramified electrodeposits (the fineness of the ramification). For example, the top right subfigure has a thin base layer and a fine ramification ( $\delta_{\text {inst }}$ and $\delta_{\text {char }}$ are both small), while the middle left subfigure has a thick base layer and a coarse ramification ( $\delta_{\text {inst }}$ and $\delta_{\text {char }}$ are both large). Lacking of a full theory for this behavior, a tentative explanation may be found in our recent stability study of the electrode deposition [40]. There we found that the base-layer thickness, defined as the instability length scale $L_{\Gamma}$ of an initially flat deposition layer, scales approximately linearly with the most unstable wavelength $\lambda_{\max }$ for a perturbation in deposition thickness along the surface. If these initial instabilities are developed into the final ramified electrodeposit, we are led to the assertion that $\delta_{\text {inst }}$ and $\delta_{\text {char }}$ might be linearly correlated. To test this assertion, we first plot the thickness $\delta_{\text {inst }}$ versus $\lambda_{\max }$ and observe the outcome. We exclude the system with $c_{0}=100 \mathrm{mM}$ and $V_{0}=$ 10 since instabilities have not yet developed in this system. The resulting plot is shown in Fig. 10(a) together with a linear fit. Although there is a good amount of scatter around the linear fit, it can be seen to capture the general trend reasonably well.

We then make a similar plot with the characteristic length scale $\delta_{\text {char }}$ of the ramified electrodeposits on the $y$ axis. To extract $\delta_{\text {char }}$, we follow the approach in Ref. [44] and calculate the so-called Minkowski dimension of each electrodeposit. In doing this we only consider the part of the electrodeposit lying between 170 and $190 \mu \mathrm{m}$ and as before we exclude the system with $c_{0}=100 \mathrm{mM}$ and $V_{0}=10$. In this work we are actually not interested in the Minkowski dimension itself, but rather in a partial result that follows from the analysis. In a range of length scales the electrodeposits appear roughly fractal, but below a certain length scale the electrodeposits are locally smooth. The length scale at which this transition occurs can be extracted from the analysis and we use this length as the characteristic length scale $\delta_{\text {char }}$ of the electrodeposit (for details see Appendix E). In Fig. 10(b) we plot $\delta_{\text {char versus }} \lambda_{\max }$ together with a linear fit of the data. While the plot suggests that $\delta_{\text {char }}$ and $\lambda_{\text {max }}$ are linearly correlated to some degree, this is a more tenuous correlation than the one between $\delta_{\text {inst }}$ and $\lambda_{\max }$.

Evidently, $\lambda_{\max }$ plays an important role for the morphology of the electrodeposits. However, $\delta_{\text {inst }}$ and $\delta_{\text {char }}$ alone are not sufficient to characterize the electrodeposits. As shown in the
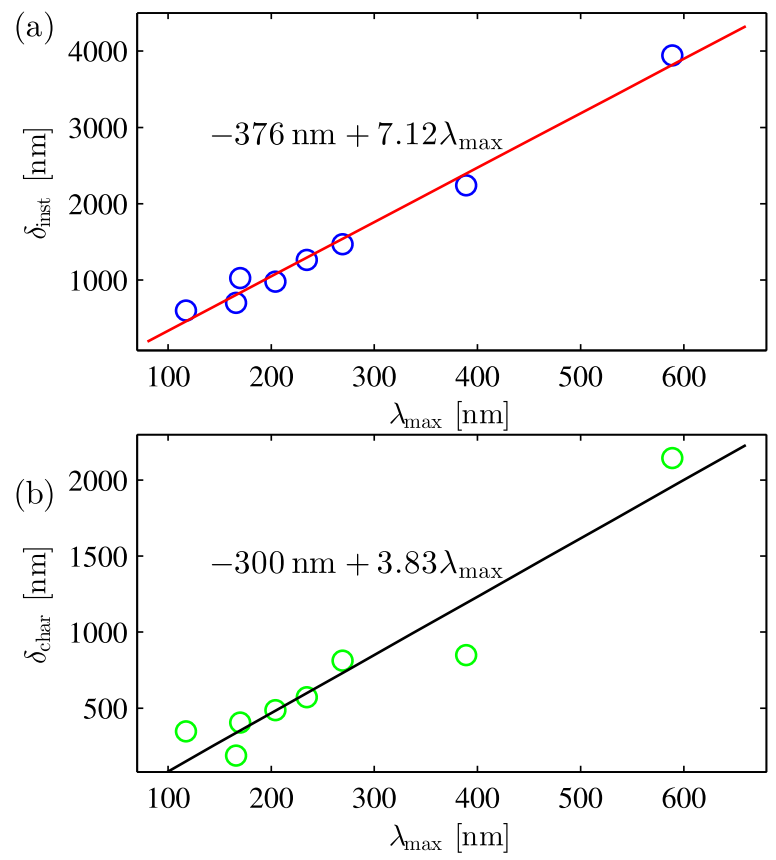

FIG. 10. (Color online) (a) Instability length scale $\delta_{\text {inst }}$ obtained from the simulations plotted versus the most unstable wavelength $\lambda_{\max }$. Also, a linear fit highlighting the roughly linear dependence is shown. (b) Characteristic length scale $\delta_{\text {char }}$ obtained from the simulations plotted versus the most unstable wavelength $\lambda_{\max }$. Also, a linear fit highlighting the roughly linear dependence is shown.

top row of Fig. 9, the characteristic length scale $\delta_{\text {char }}$ varies very little between $V_{0}=20$ and 30 . Yet the morphology still changes appreciably. The reason for this change in morphology is probably that the gradient in electrochemical potential increases near the cathode as the bias voltage is increased. The larger the electrochemical gradient is, the more the system will favor deposition at the most protruding parts of the electrodeposits. For large voltages we therefore expect long and narrow electrodeposits, whereas we expect dense branching electrodeposits for low voltages.

\section{DISCUSSION}

Our model improves on existing models in three important ways: It can treat systems at overlimiting current including the extended space-charge region, it allows for a proper reaction boundary condition, and it can be tested against results from sharp-interface stability analyses. Our model is, however, not without issues of its own. Perhaps the most apparent of these is the quasi-steady-state assumption. This assumption limits the applicability of the model to short systems, in which the diffusion time is small compared to the deposition time, as discussed in Sec. III. In principle, the phase-field models are superior to our model in this aspect since they do not have this limitation. However, it is not of practical relevance, as all of the published phase-field simulations are for systems so short that the quasi-steady-state assumption is valid anyway [25-27].

It is well known that the strong electric fields at the dendrite tips give rise to electro-osmotic velocity fields in the system [45-47]. To simplify the treatment and bring out the essential 
physics of electrodeposition, we have chosen not to include fluid dynamics and advection in our model. However, it is straightforward to include these effects (see, for instance, our previous work [36]).

One of the main advantages the sharp-interface model has over the phase-field models is that it allows for the implementation of proper reaction boundary conditions. The standard Butler-Volmer model used in this paper is a first step toward realistic reaction boundary conditions. As elaborated by Bazant in Ref. [32], there are other reaction models, such as Marcus kinetics, which might better describe the electrode reactions. Also, the standard Butler-Volmer model has the contentious assumption that the overpotential is the total potential drop over both the electrode-electrolyte interface and the Debye layer. A more realistic approach might be to model the Debye layer explicitly or include the Frumkin correction to the Butler-Volmer model [48]. Furthermore, a proper reaction expression should take the crystal structure of the material into account. There are simple ways of implementing crystal anisotropy in the surface tension term (see, for instance, Refs. $[27,49]$ ), but again, to keep the model simple, we have chosen not to include anisotropy at the present stage. Any of the above-mentioned reaction models can be easily implemented in the framework of the sharp-interface model and as such the specific Butler-Volmer model used in this work does not constitute a fundamental limitation.

More broadly, our sharp-interface model includes, or allows for the easy inclusion of, most effects that are important for electrodeposition in two dimensions. A natural next step therefore is to see how our results compare to experimental electrodeposits. Unfortunately, most such experimental data are viewed at the millimeter or centimeter scale, whereas our simulation results are at the micrometer scale. In one paper [4] the electrodeposits are probed at the micrometer scale, but the results do not make for the best comparison since the morphology of their electrodeposits was a result of adding a surface active molecule. We hope that as more experimental results become available it will be possible to perform rigorous tests of our model.

\section{CONCLUSION}

We have developed a sharp-interface model of electrodeposition, which improves on existing models in a number of ways. Unlike earlier models, our model is able to handle sharpinterface boundary conditions, such as the Butler-Volmer boundary condition, and it readily deals with regions with nonzero space-charge densities. A further advantage is that our model handles the physical problem in much the same way as done in various linear stability analyses. We can thus obtain a partial validation of our model by comparing its predictions with those of a linear stability analysis. As of now, the main weakness of our model is that it assumes a quasi-steady-state in the transport equations. For the systems studied in this paper this is a reasonable assumption since the diffusion time is small compared to the instability time. In future work we want to extend the model to the transient regime so that larger systems can be treated as well.

The main aim of this paper has been to establish the sharpinterface method, but we have also included a study of the simulated electrodeposits. An interesting observation is that there seems to be a linear correlation between the characteristic length scale of the electrodeposits and the size of the most unstable wavelength. This exemplifies a promising application of our sharp-interface model, namely, as a tool to develop a more quantitative understanding of electrodeposits and their morphology.

\section{ACKNOWLEDGMENTS}

We thank Edwin Khoo and Professor Martin Z. Bazant for valuable discussions of electrode reactions and the growth mechanisms.

\section{APPENDIX A: PHASE-FIELD MODELS OF REACTION KINETICS}

An important feature of the presented electrode-position model is that it allows for a proper nonlinear reaction model at the electrode interface. The reaction model (16) is equivalent to the expression [32]

$$
\begin{gathered}
R=i_{0}\left[e^{-\alpha n \eta_{\mathrm{t}}}-e^{(1-\alpha) n \eta_{\mathrm{t}}}\right], \\
i_{0}=k_{0} c_{+}^{1-\alpha} e^{-\bar{\gamma} \kappa},
\end{gathered}
$$

where $i_{0}$ is the exchange current density, $n$ is the number or electrons participating in the reaction, and we introduced the total overpotential $\eta_{\mathrm{t}}$ (normalized by the thermal voltage $V_{\mathrm{T}}$ ).

In phase-field models a phase-field parameter $\xi \in[0 ; 1]$ is used to distinguish between the solid phase $(\xi=1)$ and the aqueous phase $(\xi=0)$. In the interface region the phase-field parameter $\xi$ varies smoothly between 0 and 1 . To determine the dynamics of the system a free energy $F\left[\xi, c_{i}, \phi\right]$ (or grand free energy $\Omega\left[\xi, \mu_{i}, \phi\right]$ ) is introduced, which, as a function of $\xi$, interpolates between the free energy of a pure electrolyte and the free energy of a pure electrode. Using a variational approach, the ion currents can be found from the free energies

$$
\boldsymbol{J}_{i}=-D_{i} c_{i} \nabla \frac{\delta F}{\delta c_{i}}
$$

and together with the relevant conservation equations this defines the ion-transport problem.

The fundamental assumption in phase-field models of reaction kinetics is that a similar variational approach can be used to define the overpotential as a local-field quantity

$$
\eta\left[\xi, c_{i}, \phi\right] \propto \frac{\delta F}{\delta \xi} \quad \text { or } \quad \eta\left[\xi, \mu_{i}, \phi\right] \propto \frac{\delta \Omega}{\delta \xi},
$$

where we have omitted the proportionality constants. The total overpotential $\eta_{\mathrm{t}}$ is defined as the integral of the local overpotential $\eta$ over the interface region

$$
\eta_{\mathrm{t}}=\frac{1}{\delta} \int \eta\left[\xi, c_{i}, \phi\right] d x,
$$

where $\delta$ is the width of the interface region and we have assumed the system to be one dimensional. The phase-field parameter $\xi$ is then assumed to evolve according to the Butler-Volmer-like expression

$$
\partial_{t} \xi \propto\left[e^{-\alpha n \eta\left[\xi, c_{i}, \phi\right]}-e^{(1-\alpha) n \eta\left[\xi, c_{i}, \phi\right]}\right],
$$


where again we omitted the proportionality constant. The current into the electrode corresponds to the phase-field growth rate $\partial_{t} \xi$ integrated over the interface region

$$
I \propto \int \partial_{t} \xi d x \propto \int\left[e^{-\alpha n \eta\left[\xi, c_{i}, \phi\right]}-e^{(1-\alpha) n \eta\left[\xi, c_{i}, \phi\right]}\right] d x .
$$

Superficially, this expression looks fairly similar to the Butler-Volmer model (A1). However, in general, the two expressions are not identical. The reason for this is that the total overpotential occurring in Eq. (A1) is the integral over the local overpotential occurring in Eq. (A7). Thus, Eqs. (A1) and (A7) yield identical results if and only if the local overpotential $\eta$ has a form that makes it indifferent to the order in which integration and exponentiation are performed. Although this condition is satisfied for small or constant overpotentials, there is no way of guaranteeing it in general. Consequently, we expect Eq. (A7) to differ from Eq. (A1) in most cases.

Another issue with the phase-field models of reaction kinetics is that the connection between the electric potential $\phi$ and the total overpotential $\eta_{\mathrm{t}}$ is not apparent. From Ref. [32] we expect that

$$
\eta_{\mathrm{t}}=\Delta \phi-\Delta \phi_{\mathrm{eq}}
$$

where $\Delta \phi$ is the potential difference across the electrode interface and $\Delta \phi_{\text {eq }}$ is the potential difference in equilibrium. However, there does not seem to be any guarantee that this is true for phase-field reaction kinetics. In general, since most phase-field models of electrode position lack a simple sharp-interface limit [34], it is difficult to relate the parameters in a phase-field reaction model to those in the corresponding sharp-interface model.

The above objections to the phase-field reaction models do not necessarily mean that those models are wrong. After all, the Butler-Volmer model or similar nonlinear reaction models are by no means exact, so it is entirely possible that phase-field reaction models approximate the true reaction kinetics equally well. However, due to a lack of convincing reasons to believe that this is the case, the various sharp-interface reaction models remain the preferable way of modeling electrode reactions.

\section{APPENDIX B: INITIAL GROWTH}

In the initial part of the simulation the electrode is so flat that the linear stability analysis from Ref. [40] gives a good description of the growth. We parametrize the cathode position as

$$
x=X(t)+f(y, t),
$$

where $f(y, t)$ is the $y$-dependent deviation from the mean electrode position $X(t)$. According to the linear stability analysis, each mode grows exponentially in time with the growth factor $\Gamma$. After a time $t$ an initial perturbation

$$
f(y, 0)=\sum_{n=1}^{N} a_{n} e^{i k_{n} y}
$$

has therefore evolved to

$$
f(y, t)=\sum_{n=1}^{N} a_{n} e^{\Gamma_{n} t} e^{i k_{n} y} .
$$

We note that some of the growth rates $\Gamma_{n}$ can be negative. In our simulation we add new perturbations with small time intervals, which, for the purpose of this analysis, we assume to be evenly spaced. After $M$ time intervals $\Delta t$ the surface is therefore described by

$$
f(y, M \Delta t)=\sum_{m=0}^{M} \sum_{n=1}^{N} a_{n m} e^{\Gamma_{n}(M-m) \Delta t} e^{i k_{n} y} .
$$

We are interested in the average power of each mode

$$
\left\langle P_{n}\right\rangle=\left\langle\left|\sum_{m=0}^{M} a_{n m} e^{\Gamma_{n}(M-m) \Delta t}\right|^{2}\right\rangle .
$$

The coefficients are random and uncorrelated with zero mean. On average, the cross terms in the sum therefore cancel and we can simplify

$$
\begin{aligned}
\left\langle P_{n}\right\rangle & =\left\langle\sum_{m=0}^{M}\left|a_{n m}\right|^{2} e^{2 \Gamma_{n}(M-m) \Delta t}\right\rangle=\left\langle\left|a_{n}\right|^{2}\right\rangle \sum_{m=0}^{M} e^{2 \Gamma_{n}(M-m) \Delta t} \\
& =\left\langle\left|a_{n}\right|^{2}\right\rangle \frac{e^{2 \Gamma_{n}(M+1) \Delta t}-1}{e^{2 \Gamma_{n} \Delta t}-1} .
\end{aligned}
$$

The variance of the power is given as

$$
\operatorname{Var}\left(P_{n}\right)=\left\langle P_{n}^{2}\right\rangle-\left\langle P_{n}\right\rangle^{2} \text {. }
$$

The first of these terms is

$$
\begin{aligned}
\left\langle P_{n}^{2}\right\rangle & =\left\langle\left(\left|\sum_{m=0}^{M} a_{n m} e^{\Gamma_{n}(M-m) \Delta t}\right|^{2}\right)^{2}\right\rangle \\
& =e^{4 \Gamma_{n} M \Delta t}\left\langle\left(\left|\sum_{m=0}^{M} a_{n m} q^{m}\right|^{2}\right)^{2}\right\rangle
\end{aligned}
$$

where $q=e^{-\Gamma_{n} \Delta t}$. Writing out the absolute value,

$$
\left\langle P_{n}^{2}\right\rangle=e^{4 \Gamma_{n} M \Delta t}\left\langle\left(\sum_{m^{\prime}=0}^{M} \sum_{m=0}^{M} a_{n m} a_{n m^{\prime}}^{*} q^{m+m^{\prime}}\right)^{2}\right\rangle,
$$

where superscript $*$ denotes complex conjugation. Because the coefficients are uncorrelated with mean 0 , only the terms including $\left|a_{n m}\right|^{2}\left|a_{n m^{\prime}}\right|^{2}$ survive in the average of the square,

$$
\begin{aligned}
\left\langle P_{n}^{2}\right\rangle & =e^{4 \Gamma_{n} M \Delta t}\left\langle\frac{1}{2} \sum_{m^{\prime}=0}^{M} \sum_{m=0}^{M} 6\left|a_{n m}\right|^{2}\left|a_{n m^{\prime}}\right|^{2} q^{2\left(m+m^{\prime}\right)}\right\rangle \\
& =3 e^{4 \Gamma_{n} M \Delta t} \sum_{m^{\prime}=0}^{M} \sum_{m=0}^{M}\left\langle\left|a_{n m}\right|^{2}\left|a_{n m^{\prime}}\right|^{2}\right\rangle q^{2\left(m+m^{\prime}\right)} .
\end{aligned}
$$

Here the factor of 6 comes from the binomial coefficient and the factor of $\frac{1}{2}$ takes into account that the double sum counts each combination twice. Now there are two possibilities: either $m \neq m^{\prime}$ or $m=m^{\prime}$. In the first case $\left|a_{n m}\right|^{2}$ and $\left|a_{n m^{\prime}}\right|^{2}$ are uncorrelated, meaning that

$$
\left\langle\left|a_{n m}\right|^{2}\left|a_{n m^{\prime}}\right|^{2}\right\rangle=\left\langle\left|a_{n}\right|^{2}\right\rangle^{2},
$$

whereas if $m=m^{\prime}$, then

$$
\left\langle\left|a_{n m}\right|^{2}\left|a_{n m^{\prime}}\right|^{2}\right\rangle=\left\langle\left|a_{n}\right|^{4}\right\rangle
$$


This means that

$$
\begin{aligned}
\left\langle P_{n}^{2}\right\rangle= & 3 e^{4 \Gamma_{n} M \Delta t}\left\langle\left|a_{n}\right|^{2}\right\rangle^{2} \sum_{m^{\prime} \neq m}^{M} \sum_{m=0}^{M} q^{2\left(m+m^{\prime}\right)} \\
& +3 e^{4 \Gamma_{n} M \Delta t}\left\langle\left|a_{n}\right|^{4}\right\rangle \sum_{m=0}^{M} q^{4 m} \\
= & 3 e^{4 \Gamma_{n} M \Delta t}\left\langle\left|a_{n}\right|^{2}\right\rangle^{2} \sum_{m^{\prime}=0}^{M} \sum_{m=0}^{M} q^{2\left(m+m^{\prime}\right)} \\
& +3 e^{4 \Gamma_{n} M \Delta t}\left(\left\langle\left|a_{n}\right|^{4}\right\rangle-\left\langle\left|a_{n}\right|^{2}\right\rangle^{2}\right) \sum_{m=0}^{M} q^{4 m} \\
= & 3\left\langle P_{n}\right\rangle^{2} \\
& +3 e^{4 \Gamma_{n} M \Delta t}\left(\left\langle\left|a_{n}\right|^{4}\right\rangle-\left\langle\left|a_{n}\right|^{2}\right\rangle^{2}\right) \frac{q^{4(M+1)}-1}{q^{4}-1} .
\end{aligned}
$$

The variance of the power is thus given as

$$
\operatorname{Var}\left(P_{n}\right)=2\left\langle P_{n}\right\rangle^{2}+\left(\left\langle\left|a_{n}\right|^{4}\right\rangle-\left\langle\left|a_{n}\right|^{2}\right\rangle^{2}\right) \frac{e^{4 \Gamma_{n}(M+1) \Delta t}-1}{e^{4 \Gamma_{n} \Delta t}-1} .
$$

If $\Gamma_{n} \Delta t \ll 1$ we can expand the denominators of $\left\langle P_{n}\right\rangle^{2}$ and the last term. We find that they scale as $4\left(\Gamma_{n} \Delta t\right)^{2}$ and $4 \Gamma_{n} \Delta t$, respectively. In the limit $\Gamma_{n} \Delta t \ll 1$ the first term thus dominates over the second, so to a good approximation we have

$$
\begin{aligned}
& \operatorname{Var}\left(P_{n}\right) \approx 2\left\langle P_{n}\right\rangle^{2}, \\
& \operatorname{SD}\left(P_{n}\right) \approx \sqrt{2}\left\langle P_{n}\right\rangle .
\end{aligned}
$$

In the simulations the surface perturbations have the form

$$
f(y, 0)=\sum_{n=1}^{N} b_{n} h(y-n \Delta y)
$$

where

$$
h(y)= \begin{cases}1 & \text { for } 0 \leqslant y \leqslant \Delta s \\ 0 & \text { otherwise. }\end{cases}
$$

We take the absolute square of $f(y, 0)$ given as both Eqs. (B2) and (B17) and integrate over the domain to obtain

$$
\begin{aligned}
\int_{0}^{W}|f(y, 0)|^{2} d y & =\sum_{n=1}^{N}\left|b_{n}\right|^{2} \int_{0}^{W}|h(y-n \Delta y)|^{2} d y \\
= & \Delta s \sum_{n=1}^{N}\left|b_{n}\right|^{2} \\
\int_{0}^{W}|f(y, 0)|^{2} d y & =\sum_{n=1}^{N}\left|a_{n}\right|^{2} \int_{0}^{W}\left|e^{i k_{n} y}\right|^{2} d y \\
& =W \sum_{n=1}^{N}\left|a_{n}\right|^{2}
\end{aligned}
$$

The mean square of $b_{n}$ is thus related to the mean square of $a_{n}$ as

$$
\left\langle\left|a_{n}\right|^{2}\right\rangle=\frac{\Delta s}{W}\left\langle\left|b_{n}\right|^{2}\right\rangle=\frac{1}{N}\left\langle\left|b_{n}\right|^{2}\right\rangle .
$$

From Eq. (37) we have that

$$
\left\langle\left|b_{n}\right|^{2}\right\rangle=a^{6} \frac{J_{+} \Delta t}{\Delta h \Delta s} .
$$

Inserting in Eq. (B6) we find

$$
\begin{aligned}
\left\langle P_{n}\right\rangle & =\frac{1}{N} a^{6} \frac{J_{+} \Delta t}{\Delta h \Delta s} \frac{e^{2 \Gamma_{n}(M+1) \Delta t}-1}{e^{2 \Gamma_{n} \Delta t}-1} \\
& =a^{6} \frac{J_{+} \Delta t}{\Delta h W} \frac{e^{2 \Gamma_{n}\left(t_{\mathrm{tot}}+\Delta t\right)}-1}{e^{2 \Gamma_{n} \Delta t}-1},
\end{aligned}
$$

which is seen to be independent of the bin size $\Delta s$. We also introduced the total time $t_{\text {tot }}=M \Delta t$. In a consistent scheme the power spectrum should of course only depend on the total time and not on the size $\Delta t$ of the time steps. For small values of $\Gamma_{n} \Delta t$ we can expand the denominator and neglect the $\Delta t$ in the nominator,

$$
\left\langle P_{n}\right\rangle \approx a^{6} \frac{J_{+}}{2 \Delta h W \Gamma_{n}}\left[e^{2 \Gamma_{n} t_{\mathrm{tot}}}-1\right] .
$$

So, as long as $2 \Gamma_{n} \Delta t \ll 1$, the power spectrum does not depend on the size of the time step.

For larger values of $2 \Gamma_{n} \Delta t$ the power spectrum does depend on the size of the time step. However, as long as $2 \Gamma_{n} \Delta t \lesssim 1$, we do not expect the overall morphology of the electrode to have a significant dependence on the time step.

\section{APPENDIX C: TEST OF THE DOMAIN REDUCTION}

In the numerical simulations we exploit the fact that the electrode growth mainly occurs near the dendrite tips. This

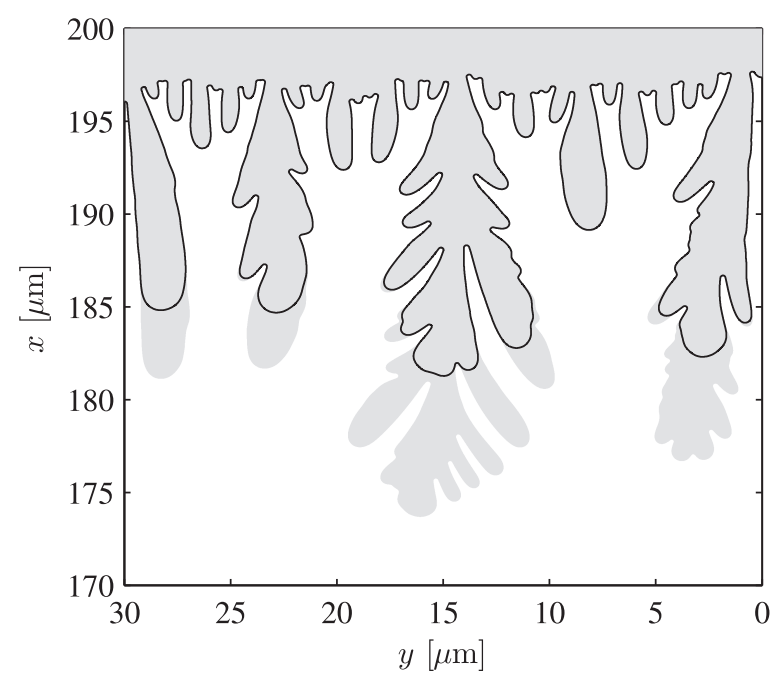

FIG. 11. Electrode interface at two different time steps for $c_{0}=$ $1 \mathrm{mM}$ and $V_{0}=10$. In this simulation no part of the domain was rendered passive at any time. The gray area indicates the electrode after $30 \mathrm{~h}$ and $22 \mathrm{~min}$ and the black line indicates the electrode interface after $24 \mathrm{~h}$ and $2 \mathrm{~min}$. In the upper part of the domain the electrode interface is virtually unchanged between the two times. 


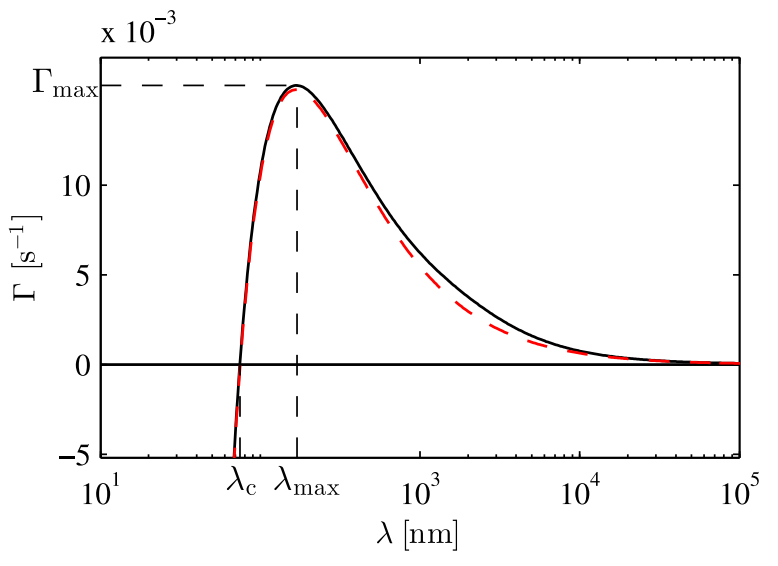

FIG. 12. (Color online) Instability growth rate $\Gamma$ plotted versus perturbation wavelength $\lambda$. The solid (black) line is the numerical result and the dashed (red) line is the analytical result. The critical wavelength $\lambda_{c}$ and the most unstable wavelength $\lambda_{\max }$ are indicated in the figure. The parameter values used in the model are those in Table I as well as $c_{0}=10 \mathrm{mM}, L=100 \mu \mathrm{m}$, and $V_{0}=30$.

allows us to remove part of the domain (the passive region) from the simulations. To verify that we do not significantly affect the growth, by rendering part of the domain passive, we have carried out a simulation without a passive region. The gray domain in Fig. 11 indicates the electrode after deposition for $30 \mathrm{~h}$ and $22 \mathrm{~min}$ in a system with $c_{0}=10 \mathrm{mM}$ and $V_{0}=10$. The black line indicates the electrode interface after deposition for $24 \mathrm{~h}$ and $2 \mathrm{~min}$. It can be seen that a big part of the domain is virtually unchanged between the two times. Therefore, it would not have changed the results appreciably if we had rendered part of the domain passive.

\section{APPENDIX D: STABILITY ANALYSIS}

In Ref. [40] we investigated the stability of the electrode interfaces under perturbations of varying wavelengths. We found numerical and analytical result for the instability growth rate

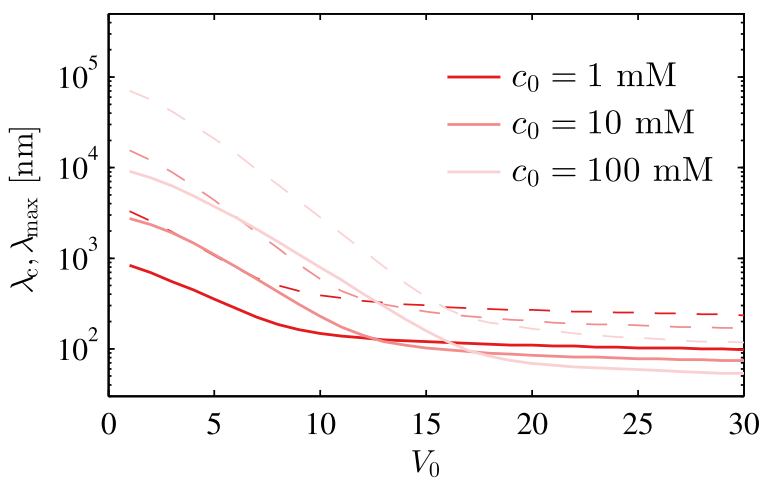

FIG. 13. (Color online) Critical wavelength $\lambda_{\mathrm{c}}$ (solid line) and the most unstable wavelength $\lambda_{\max }$ (dashed line) plotted versus $V_{0}$. In addition to the values listed in Table I, the parameter values used are $L=100 \mu \mathrm{m}$ and $c_{0}=\{1 \mathrm{mM}, 10 \mathrm{mM}, 100 \mathrm{mM}\}$.

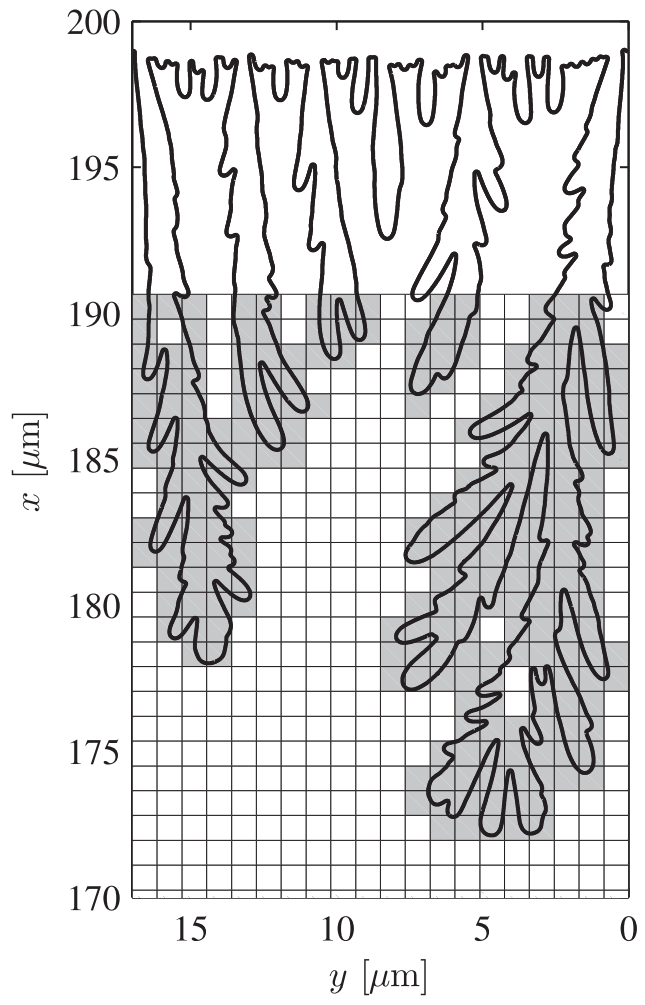

FIG. 14. Illustration of the box-counting method leading to the Minkowski dimension for the electrodeposit obtained for $c_{0}=$ $10 \mathrm{mM}$ and $V_{0}=20$. The boxes that cover part of the deposit perimeter are shown in gray and the remaining boxes are shown in white. In this example the grid size is $\epsilon=0.85 \mu \mathrm{m}$ and the number of boxes it takes to cover the perimeter is $N(\epsilon)=234$.

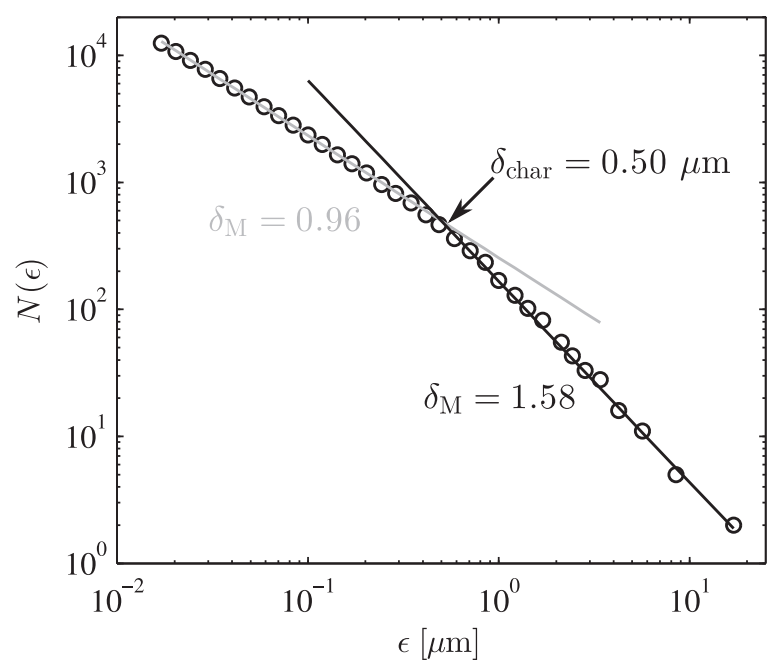

FIG. 15. Number $N(\epsilon)$ of boxes it takes to cover the electrodeposit plotted vs the box side length $\epsilon$. A linear fit is shown in each of the two approximately linear regions and the Minkowski dimension in each region is indicated. The crossing point between the linear fits is marked by an arrow and the characteristic dimension $\delta_{\text {char }}=0.50 \mu \mathrm{m}$ is calculated based on this crossing point. 
$\Gamma$, the critical wavelength $\lambda_{\mathrm{c}}$, and the most unstable wavelength

$\lambda_{\text {max }}$. In Fig. 12 the instability growth rate $\Gamma$ is plotted versus perturbation wavelength for $c_{0}=10 \mathrm{mM}, L=100 \mu \mathrm{m}$, and $V_{0}=30$. The critical wavelength $\lambda_{\mathrm{c}}$ and the most unstable wavelength $\lambda_{\max }$ are indicated in the plot. In Fig. 13 we plot $\lambda_{\mathrm{c}}$ and $\lambda_{\max }$ versus $V_{0}$ for $c_{0}=\{1 \mathrm{mM}, 10 \mathrm{mM}, 100 \mathrm{mM}\}$ and $L=100 \mu \mathrm{m}$.

\section{APPENDIX E: CHARACTERISTIC LENGTH SCALE}

To find the characteristic length scale $\delta_{\text {char }}$ of the ramified electrodeposits we follow Ref. [44] and use the box-counting method to calculate the Minkowski dimension of the deposit perimeter. As a first step in calculating the Minkowski dimension, we place a square grid with side length $\epsilon$ over each deposit and count the number $N(\epsilon)$ of boxes it takes to completely cover the perimeter of the part of the deposit lying between $x=170$ and $190 \mu \mathrm{m}$. An example is shown in Fig. 14.
For a proper fractal geometry, the Minkowski dimension is defined as

$$
\delta_{\mathrm{M}}=-\lim _{\epsilon \rightarrow 0} \frac{\ln [N(\epsilon)]}{\ln (\epsilon)} .
$$

The electrodeposits we are investigating are not fractal at all length scales, but in a range of length scales, we can calculate an approximate Minkowski dimension as the negative slope in a $\ln [N(\epsilon)]$ vs $\ln (\epsilon)$ plot. In Fig. 15 such a plot is shown together with linear fits in each of the two approximately linear regions. The Minkowski dimension at small $\epsilon$ is nearly unity, indicating that the deposit perimeter is locally smooth at this length scale. For larger values of $\epsilon$ the Minkowski dimension deviates from unity because the deposit is approximately fractal in this size range. At the transition point between these two regions is the smallest length scale, which is related to the morphology of the electrodeposit. We denote this length scale by the characteristic length $\delta_{\text {char }}$. Technically, we define $\delta_{\text {char }}$ as the point where the linear fits from each region cross each other, as indicated in Fig. 15.
[1] V. Fleury, W. Watters, L. Allam, and T. Devers, Nature (London) 416, 716 (2002).

[2] M. Rosso, Electrochim. Acta 53, 250 (2007).

[3] J. W. Gallaway, A. M. Gaikwad, B. Hertzberg, C. K. Erdonmez, Y.-C. K. Chen-Wiegart, L. A. Sviridov, K. Evans-Lutterodt, J. Wang, S. Banerjee, and D. A. Steingart, J. Electrochem. Soc. 161, A275 (2014).

[4] T.-H. Lin, C.-W. Lin, H.-H. Liu, J.-T. Sheu, and W.-H. Hung, Chem. Commun. 47, 2044 (2011).

[5] M. Park, X. Zhang, M. Chung, G. B. Less, and A. M. Sastry, J. Power Sources 195, 7904 (2010).

[6] B. Scrosati and J. Garche, J. Power Sources 195, 2419 (2010).

[7] J. M. Tarascon and M. Armand, Nature (London) 414, 359 (2001).

[8] M. Winter and R. J. Brodd, Chem. Rev. 104, 4245 (2004).

[9] J.-H. Han, E. Khoo, P. Bai, and M. Bazant, Sci. Rep. 4, 7056 (2014).

[10] H.-C. Shin, J. Dong, and M. Liu, Adv. Mater. 15, 1610 (2003).

[11] O. Devos, C. Gabrielli, L. Beitone, C. Mace, E. Ostermann, and H. Perrot, J. Electroanal. Chem. 606, 75 (2007).

[12] J.-N. Chazalviel, Phys. Rev. A 42, 7355 (1990).

[13] M. Z. Bazant, Phys. Rev. E 52, 1903 (1995).

[14] L. Sundstrom and F. Bark, Electrochim. Acta 40, 599 (1995).

[15] G. Gonzalez, M. Rosso, and E. Chassaing, Phys. Rev. E 78, 011601 (2008).

[16] K. Nishikawa, E. Chassaing, and M. Rosso, J. Electrochem. Soc. 160, D183 (2013).

[17] P. Trigueros, J. Claret, F. Mas, and F. Sagues, J. Electroanal. Chem. 312, 219 (1991).

[18] G. Kahanda and M. Tomkiewicz, J. Electrochem. Soc. 136, 1497 (1989).

[19] N. Nikolic, K. Popov, L. Pavlovic, and M. Pavlovic, Surf. Coat. Technol. 201, 560 (2006).
[20] C. Lambert, P. Lauque, J.-L. Seguin, G. Albinet, M. Bendahan, J.-M. Debierre, and P. Knauth, Chem. Phys. Chem. 3, 107 (2002).

[21] T. A. Witten and L. M. Sander, Phys. Rev. B 27, 5686 (1983).

[22] T. A. Witten and L. M. Sander, Phys. Rev. Lett. 47, 1400 (1981).

[23] J. E. Guyer, W. J. Boettinger, J. A. Warren, and G. B. McFadden, Phys. Rev. E 69, 021603 (2004).

[24] J. E. Guyer, W. J. Boettinger, J. A. Warren, and G. B. McFadden, Phys. Rev. E 69, 021604 (2004).

[25] Y. Shibuta, Y. Okajima, and T. Suzuki, Sci. Technol. Adv. Mater. 8, 511 (2007).

[26] L. Liang and L. Chen, Appl. Phys. Lett. 105, 263903 (2014).

[27] D. A. Cogswell, Phys. Rev. E 92, 011301 (2015).

[28] D. Ely, A. Jana, and R. E. Garcia, J. Power Sources 272, 581 (2014).

[29] D. A. Cogswell and M. Z. Bazant, ACS Nano 6, 2215 (2012).

[30] W. H. Smyrl and J. Newman, Trans. Faraday Soc. 63, 207 (1967).

[31] J. Dukovic, IBM J. Res. Dev. 34, 693 (1990).

[32] M. Z. Bazant, Acc. Chem. Res. 46, 1144 (2013).

[33] J. Deng, G. J. Wagner, and R. P. Muller, J. Electrochem. Soc. 160, A487 (2013).

[34] L. Liang, Y. Qi, F. Xue, S. Bhattacharya, S. J. Harris, and L. Q. Chen, Phys. Rev. E 86, 051609 (2012).

[35] C. P. Nielsen and H. Bruus, Phys. Rev. E 89, 042405 (2014).

[36] C. P. Nielsen and H. Bruus, Phys. Rev. E 90, 043020 (2014).

[37] V. Fleury and D. Barkey, Europhys. Lett. 36, 253 (1996).

[38] C. Leger, L. Servant, J. L. Bruneel, and F. Argoul, Physica A 263, 305 (1999).

[39] C. Leger, J. Elezgaray, and F. Argoul, Phys. Rev. E 61, 5452 (2000).

[40] C. P. Nielsen and H. Bruus, arXiv:1505.07571.

[41] M. M. Gregersen, M. B. Andersen, G. Soni, C. Meinhart, and H. Bruus, Phys. Rev. E 79, 066316 (2009).

[42] D. R. Lide, CRC Handbook of Chemistry and Physics, 91st ed., edited by W. M. Haynes (CRC, Boca Raton, 2010). 
[43] D. R. Turner and G. R. Johnson, J. Electrochem. Soc. 109, 798 (1962).

[44] A. Genau, A. Freedman, and L. Ratke, J. Cryst. Growth 363, 49 (2013).

[45] V. Fleury, J.-N. Chazalviel, and M. Rosso, Phys. Rev. Lett. 68, 2492 (1992).
[46] V. Fleury, J. Kaufman, and B. Hibbert, Phys. Rev. E 48, 3831 (1993).

[47] J. M. Huth, H. L. Swinney, W. D. McCormick, A. Kuhn, and F. Argoul, Phys. Rev. E 51, 3444 (1995).

[48] M. van Soestbergen, Russ. J. Electrochem. 48, 570 (2012).

[49] R. Kobayashi, Physica D 63, 410 (1993). 